\title{
Novel transverse emittance measurements for electron cooling characterization
}

\author{
J. R. Hunt $\odot,{ }^{*}$ J. Resta-López, and C. P. Welsch \\ Department of Physics, University of Liverpool, L69 3BX, United Kingdom \\ and The Cockcroft Institute, Daresbury, WA4 4AD, United Kingdom \\ C. Carli, B. Dupuy, and D. Gamba \\ CERN, CH-1211 Geneva-23, Switzerland
}

(Received 10 November 2019; accepted 20 February 2020; published 3 March 2020)

\begin{abstract}
The extra low energy antiproton ring (ELENA) finished commissioning before the start of CERN's second long shutdown in December 2018, successfully providing beams to a new experimental zone. In 2021, ELENA will begin distributing cooled $100 \mathrm{keV}$ antiproton beams to all antimatter experiments. To counteract beam blowup due to deceleration, ELENA will employ the use of an electron cooler. Measurements under similar circumstances, such as at the antiproton decelerator at CERN, have shown electron cooling causing non-Gaussian beam profiles. This effect, combined with nonzero dispersion at the location of the scraper in ELENA, presents new challenges in the use of ELENA's scraper to determine the emittance during the deceleration cycle. Two new scraper algorithms have been developed and used to show the first evidence of significant electron cooling in ELENA, at 650 and $100 \mathrm{keV}$ energy plateaus. The algorithms are capable of estimating the longitudinal momentum spread of the beams and accurately determining emittances for non-Gaussian beams in dispersive regions. Additionally, utilizing combinations of measurements from opposing scraper blades, additional information on the beam's evolution is presented, suggesting a correlation between the emittance and longitudinal momentum offset of individual particles. Finally, considerations for further studies in ELENA and similar machines are presented.
\end{abstract}

DOI: 10.1103/PhysRevAccelBeams.23.032802

\section{INTRODUCTION}

It is essential during the commissioning and operation of any modern accelerator facility to measure and track the emittance of the propagating beams. In particular, for a synchrotron, the transverse emittance provides information on beam quality at various stages of the acceleration cycle. In the antiproton decelerator (AD) [1,2] and the newly commissioned extra low energy antiproton ring (ELENA) [3-5], antiproton beams experience an adiabatic blowup of emittance during deceleration, which must be monitored and counteracted before extraction to experiments. Additionally, at low energies, collective effects such as intrabeam scattering (IBS) [6-9] become significant and contribute to emittance growth. Both machines employ the use of an electron cooler to reduce emittances during intermediate and extraction energy plateaus [10]. The

\footnotetext{
*james.hunt@cern.ch
}

Published by the American Physical Society under the terms of the Creative Commons Attribution 4.0 International license. Further distribution of this work must maintain attribution to the author(s) and the published article's title, journal citation, and DOI. nominal parameters for ELENA's electron cooler may be found in Table I.

In order to measure the emittance at a specific time during the machine's cycle, a scraping device has been successfully employed in the $\mathrm{AD}$ [11], from injection energy $2.8 \mathrm{GeV}$ down to an extraction energy of $5.3 \mathrm{MeV}$. The scraper has been shown to effectively probe these low-energy, low-intensity $\left(\mathrm{N}_{p} \approx 5 \times 10^{7}\right)$ beam

TABLE I. Nominal electron cooler parameters for ELENA.

\begin{tabular}{|c|c|c|}
\hline & $650 \mathrm{keV}$ & $100 \mathrm{keV}$ \\
\hline Electron beam energy $(\mathrm{eV})$ & 355 & 55 \\
\hline Electron current $(\mathrm{mA})$ & 5 & 2 \\
\hline Electron beam density $\left(\mathrm{m}^{-3}\right)$ & $1.38 \times 10^{12}$ & $1.41 \times 10^{12}$ \\
\hline$B_{\text {gun }}(G)$ & \multicolumn{2}{|c|}{1000} \\
\hline $\mathrm{B}_{\text {drift }}(\mathrm{G})$ & \multicolumn{2}{|c|}{100} \\
\hline Expansion factor & \multicolumn{2}{|c|}{10} \\
\hline Cathode radius (mm) & \multicolumn{2}{|c|}{8} \\
\hline Electron beam radius (mm) & \multicolumn{2}{|c|}{25} \\
\hline$\beta_{x}, \beta_{y}, D_{x}(\mathrm{~m})$ & \multicolumn{2}{|c|}{$2.103,2.186,1.498$} \\
\hline Flange-to-flange length (mm) & \multicolumn{2}{|c|}{2330} \\
\hline Drift solenoid length (mm) & \multicolumn{2}{|c|}{1000} \\
\hline Effective length (mm) & \multicolumn{2}{|c|}{700} \\
\hline
\end{tabular}


distributions and so was also chosen as the primary emittance diagnostic for ELENA, which has an extraction energy of $100 \mathrm{keV}$. The basic principle of a moving scraper blade measurement is as follows: The beam is destructively intercepted by a metallic scraper blade, which moves slowly with respect to the revolution frequency. As the beam is intercepted, a shower of secondary particles is generated. If the scraper blade moves slowly enough, the corresponding shower intensity is proportional to the maximum oscillation amplitude density of the particles at the position of the scraper blade. By detecting this shower and observing how its intensity evolves as a function of the scraper blade position, it is possible to gain an insight into the transverse beam profile distribution. The emittance of the beam can be estimated by assuming the necessary lattice functions at the scraper's location.

In the case of the $\mathrm{AD}$ and ELENA, secondary signals on scintillators outside the beam pipe are dominated by the pions generated from antiproton annihilations within the scraper blade. The secondary signal given as a function of the scraper blade position may be summed to produce a cumulative density function (CDF), $F_{ \pm}$. In the AD system, the CDF is used to obtain an estimate for the distance between the region containing $95 \%$ of the beam and beam core $(0 \%$ intensity in $\mathrm{CDF}), \sigma_{95}$. This quantity is simply converted to the $95 \%$ emittance with $\epsilon_{95}=\sigma_{95}^{2} / \beta$, where $\beta$ is the transverse beta function at the scraper corresponding to the scraping direction, $x$ or $y$.

In ELENA, there is no region with zero dispersion, and, as such, dispersive effects at the scraper unavoidably convolute the CDF. Additionally, non-Gaussian transverse beam distributions resulting from deceleration and electron cooling present further challenges in extracting accurate emittance estimates from obtained signals.

Two novel scraper algorithms have been developed to surmount the unique challenges presented by ELENA, and, through detailed simulations, they have been shown to accurately reconstruct the statistical rms emittance, $\epsilon_{r m s}$, in a range of conditions [12]. The statistical rms emittance is defined as

$$
\epsilon_{r m s}=\langle J\rangle \equiv \frac{1}{2}\langle A\rangle^{2},
$$

where $\langle\cdots\rangle$ denotes the expectation value of the quantity in the parentheses, $J$ is the angle action variable, and $A$ is the amplitude of the particles in phase space.

One algorithm utilizes a line fit to the data, assuming the beam may be approximated as Gaussian, and is also capable of reconstructing the momentum spread of the beam by taking it as a second free parameter during fitting. The second algorithm requires two separate scraper measurements from opposite directions and is capable of reconstructing the emittance for an arbitrarily shaped beam in a region of nonzero dispersion. ELENA is equipped with two arms, each housing a windowlike configuration through which the beam passes. With the two edges perpendicular to arm motion per arm, it is possible to scrape from four transverse directions.

In this paper, scraper measurements taken during ELENA commissioning at the end of 2018 are presented and analyzed using these new algorithms. The measurements aim to showcase the utility of the algorithms and also to provide the first evidence of effective electron cooling within ELENA. Additionally, the two-scan algorithm provides an insight into a correlation between the emittance and momentum offset of beam particles.

\section{MEASUREMENTS}

The measurements have been carried out in all four transverse directions, at three times along ELENA's cycle, as schematically represented on a typical ELENA cycle plot in Fig. 1. Two sets of measurements were made at the $650 \mathrm{keV}$ intermediate energy plateau at separate cycle times during electron cooling $(t=7.8 \mathrm{~s}$ and $t=14.5 \mathrm{~s}$, respectively), and two sets during the $100 \mathrm{keV}$ extraction energy plateau, both at the same cycle time $(3.9 \mathrm{~s}$ along the plateau), with and without electron cooling. Some scraper measurements were repeated in order to check reproducibility and obtain uncertainties. All measurements were made with rf systems off, as the algorithms require a coasting beam. Additionally, Schottky measurements [13] to determine the rms momentum spread at the corresponding times were performed. The rms momentum spread is needed as an input for the two-scan algorithm.

The underlying equation for the two-scan algorithm may be expressed as

$$
\epsilon_{r m s}=\frac{1}{4 \beta}\left[\sigma_{+}^{2}+\sigma_{-}^{2}+\frac{\left(\bar{x}_{+}-\bar{x}_{-}\right)^{2}}{2}\right]-\frac{D^{2} \sigma_{\delta}^{2}}{2 \beta},
$$

where $D$ is the dispersion in the plane of scraping, $\beta$ is the beta function of the same plane, and $\sigma_{\delta}$ is the rms momentum spread at the time of the measurement. By taking the derivative of the $\mathrm{CDF}$, introduced earlier, a probability density function (PDF) $f_{ \pm}$is obtained. The

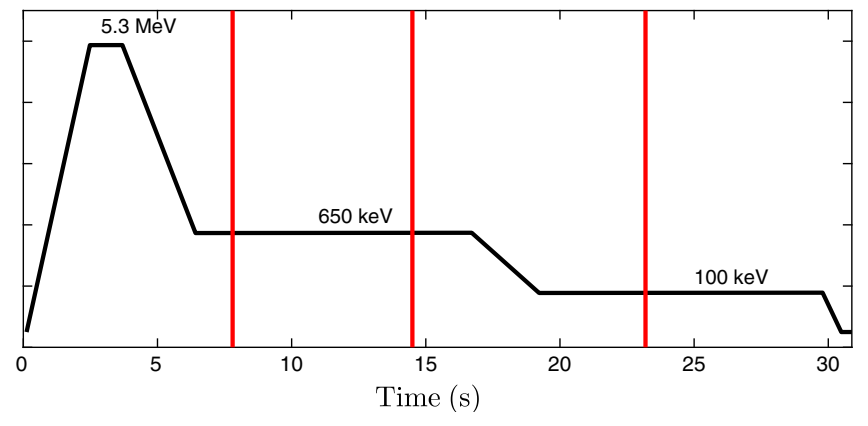

FIG. 1. Cycle times at which sets of scraper measurements were performed, marked with vertical red lines. 
subscripts + and - refer to the direction in which the beam is being scraped (e.g., from positive or negative $x$ ). The values $\sigma_{ \pm}$and $\bar{x}_{ \pm}$are the rms and mean of $f_{ \pm}$, respectively. This equation is first introduced and derived in Ref. [12], along with a detailed description of the single-scan line-fit method, which assumes a Gaussian beam, and can estimate the momentum spread.

\section{A. Quantities obtained without scraping}

It can be seen in Eq. (2) that there are several inputs required for the emittance, including three $\left(\sigma_{\delta}, \beta\right.$, and $\left.D\right)$ that do not come from scraper measurements directly. Values for the horizontal and vertical beta functions $\beta_{x}$ and $\beta_{y}$, respectively, and the horizontal dispersion $D_{x}$ were calculated based on the optical settings around the ring at the time of measurement. The values at the scraper were determined to be $\beta_{x}=3.2 \mathrm{~m}, \beta_{y}=2.97 \mathrm{~m}$, and $D_{x}=1.38 \mathrm{~m}$.

Uncertainties in these measurements were calculated using a MAD-X [14] model of the optics with incremental field strength errors to observe trends in resultant errors. The horizontal beta was seen to be comparatively sensitive, and uncertainties in measurements corresponding to $\pm 5 \%$ field strength errors were determined and propagated to final emittance errors.

In order to calculate the rms momentum spread, Schottky analysis of the signal seen on a transverse pickup was used. Schottky data were available only for the intermediate $650 \mathrm{keV}$ plateau at the time of measurements. A spectral density distribution is given by a spectrum analyzer, scanning around the tenth harmonic of the revolution frequency, $371 \mathrm{kHz}$. The resultant distribution can be seen in Fig. 2, including signals given during rebunching on the

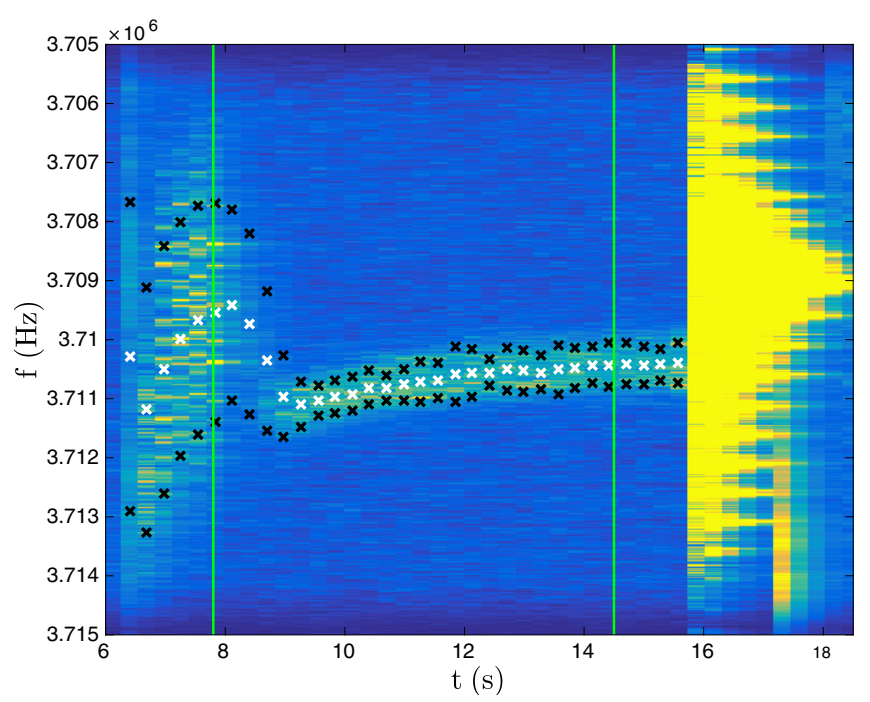

FIG. 2. The spectral density distribution obtained from Schottky measurements in the presence of electron cooling during the $650 \mathrm{keV}$ energy plateau. The black and white crosses represent fitted $\Delta f_{h}$ and $f_{h}$ values, respectively. Scraper measurement times are highlighted by vertical green lines. right-hand side. Each time slice of these spectra was evaluated using

$$
\sigma_{\delta}=-\frac{1}{\eta} \frac{\Delta f_{h}}{f_{h}}
$$

where $\eta$ is the phase slip factor, $f_{h}$ is the central frequency of the signal peak, and $\Delta f_{h}$ is the rms width of the peak. Similarly to the optical quantities at the scraper, $\eta$ was calculated based the strength settings of the elements around the ring.

The values of $\Delta f_{h}$ and $f_{h}$ given by numerical analysis for each time slice along the intermediate plateau are also shown with crosses on Fig. 2. These values were then converted to rms momentum spreads, the time evolution of which may be observed in Fig. 3. A clear and rapid reduction to an equilibrium plateau can be observed due to effective electron cooling. Values of $1.1( \pm 0.16) \times 10^{-3}$ and $0.22( \pm 0.03) \times 10^{-3}$ were determined for $t=7.8 \mathrm{~s}$ and $t=14.5 \mathrm{~s}$, respectively, and were used as input parameters for the two-scan algorithm analysis.

\section{B. Quantities obtained through scraping}

Raw data corresponding to a secondary signal per time were obtained from scintillators and, using scraper time $\left(t_{s}\right)$ and position $\left(x_{s}, y_{s}\right)$ readings, converted to signal per position measurements. Here, cuts in data on either side of a clear secondary beam signal (typically between $\pm 25 \mathrm{~mm}$ ) were made to prevent background noise such as injection losses appearing as high-amplitude particles. Despite their relatively low intensity, such erroneous signals may heavily skew resultant quantities due to their perceived high transverse positions, and so such cuts are essential.

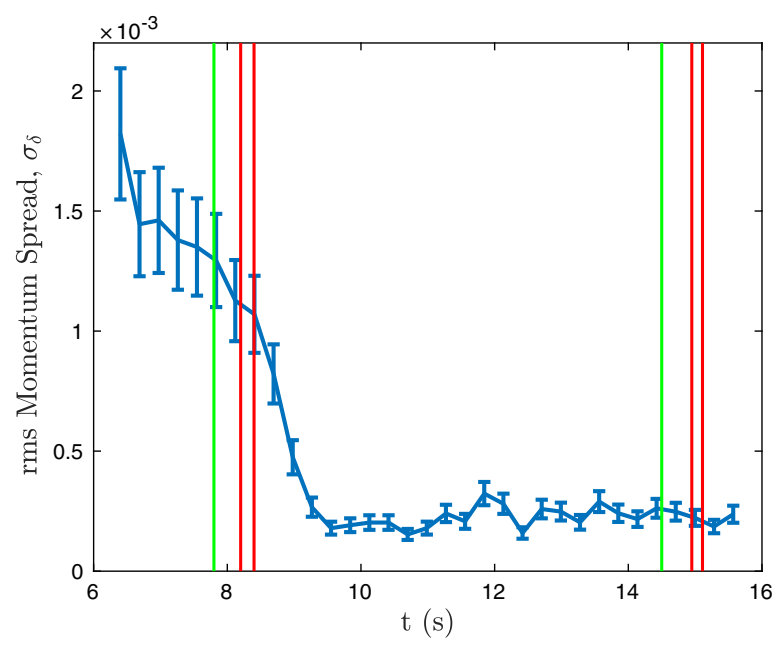

FIG. 3. Time evolution of the rms longitudinal momentum spread, $\sigma_{\delta}$, during the $650 \mathrm{keV}$ energy plateau in the presence of electron cooling. Green and red vertical lines highlight the activation time of the scraper and the time window corresponding to the signal seen on scintillators, respectively. 
Once secondary signal per time measurements were obtained, data were cumulatively summed and then normalized to unity in order to give a $\mathrm{CDF}, F_{ \pm}\left(x_{s}\right)$ (similarly for $y_{s}$, although here we consider only horizontal measurements for brevity). It is worth pointing out that through this normalization the relative absolute intensities of detector signals become irrelevant (provided they are well distinguished from noise). The PDF is then obtained through differentiation by finite difference approximations: $f_{ \pm}\left(x_{s}\right)=\mathrm{d} F_{ \pm}\left(x_{s}\right) / \mathrm{d} x_{s}$. This distribution is then finally converted to input quantities for the algorithm from

$$
\begin{aligned}
& \bar{x}_{ \pm}=\int_{-\infty}^{+\infty} f_{ \pm}\left(x_{s}\right) x_{s} \mathrm{~d} x_{s}, \\
& \sigma_{ \pm}^{2}=\int_{-\infty}^{+\infty} f_{ \pm}\left(x_{s}\right)\left(x_{s}-\bar{x}_{ \pm}\right)^{2} \mathrm{~d} x_{s},
\end{aligned}
$$

integrating the data with finite difference methods.

Repeat scraper measurements were performed to check for shot-to-shot fluctuations. The momentum-dependent closed orbit of the beam may be extracted from measurements using

$$
x_{0}+D \bar{\delta}=\frac{\bar{x}_{+}+\bar{x}_{-}}{2},
$$

where $x_{0}$ is the horizontal closed orbit of the beam, $\bar{\delta}$ denotes the mean momentum offset of the beam, and similarly for measurements in the vertical plane, $y$. This quantity showed no significant change between shots in both planes.

The measurements also served to potentially expose errors in scraper position readings between shots. Such fluctuations or errors would be significant, since this method requires the combination of two separate scraper measurements. Comparisons in the calculated means of PDFs, $\Delta \bar{x}_{ \pm}$ and $\Delta \bar{y}_{ \pm}$, obtained for repeat horizontal and vertical measurements yielded differences of 0.03 and $0.04 \mathrm{~mm}$, respectively, and so the effect was deemed negligible.

\section{ANALYSIS}

\section{A. Intermediate $650 \mathrm{keV}$ energy plateau}

Since measurements are simplified in the vertical plane due to zero vertical dispersion, this was the best place to begin testing the algorithms. Setting $\sigma_{\delta}$ to zero and hence removing the final term in Eq. (2), $\epsilon_{y}$ was determined to be $1.59( \pm 0.02)$ and $1.15( \pm 0.02) \mathrm{mm} \mathrm{mrad}$ for measurements at 7.8 and $14.5 \mathrm{~s}$, respectively, equating to an emittance reduction of $28( \pm 2) \%$ and confirming transverse cooling has taken place. Reconstructed momentum-dependent closed orbit values showed no significant change after cooling, as expected. An offset from $y_{0}$ could be explained due to a calibration offset in the scraper window position and may be investigated after CERN's second long shutdown. Such a calibration offset would not affect accuracy of the emittance results, since a single arm scrapes in both directions with opposing blades attached in a windowlike configuration.

Figure 4 shows the CDFs corresponding to both pairs of measurements. A shift in the CDFs at $14.5 \mathrm{~s}$ toward the beam core is indicative of the reduced emittance after some exposure of the beam to electron cooling. Using a custom PYTHON [15] Monte Carlo simulation tool, two pairs of CDFs have been generated for Gaussian distributions, with emittances corresponding to the reconstructed emittance values, and similarly for the closed orbits. Comparing the two cases, it can be observed that the Gaussian approximation better fits the CDFs at $t=7.8 \mathrm{~s}$. A more densely populated core with wider tails at $t=14.5 \mathrm{~s}$ suggests that indeed electron cooling has been more effective on the core of the beam as predicted, leading to non-Gaussian beam distributions.

The single-scan Gaussian line-fit algorithm was also used on these results and compared with the two-scan results as seen in Table II. While there appears to be a consistent underestimation of the emittance for the line-fit method, it can be seen that there is a more significant disagreement between the results after some time exposed to electron cooling, further supporting that the beam has become less Gaussian. This comparison may also serve to provide an insight into how sensitive the line-fit algorithm is to the transverse profile of the beam.

Horizontal scraper measurements at this energy were also analyzed, this time using the values of momentum spread, $1.1( \pm 0.16) \times 10^{-3}$ and $0.22( \pm 0.03) \times 10^{-3}$ for $t=$ $7.8 \mathrm{~s}$ and $t=14.5 \mathrm{~s}$, respectively, presented in Sec. II A. For the same respective times, the two-scan algorithm returned values of $3.6( \pm 0.27)$ and $0.7( \pm 0.05) \mathrm{mm} \mathrm{mrad}$, showing a much more significant decrease of $81( \pm 10) \%$,

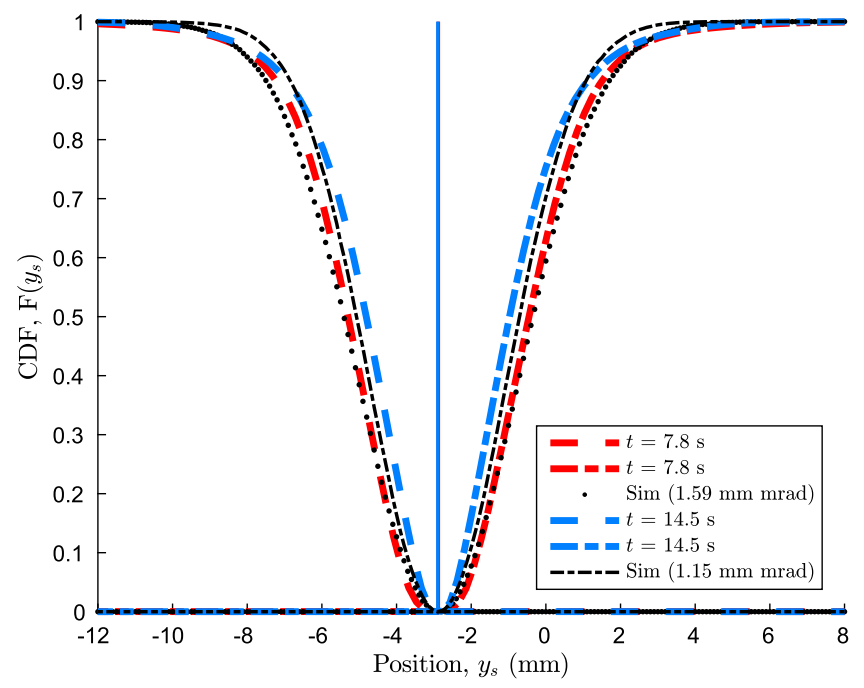

FIG. 4. Measured CDFs from vertical scraper scans are represented by colored dashed lines. Black dashed lines highlight corresponding simulations, while two (overlaying) vertical solid lines mark the calculated closed orbit. 
TABLE II. Comparison of results from the single-scan Gaussian algorithm with the two-scan method. One-scan T and B refer to scraping from the top and bottom, respectively. $\Delta \epsilon_{y}$ refers to the difference from the two-scan results.

\begin{tabular}{lcccccc}
\hline \hline & \multicolumn{2}{c}{$t=7.8 \mathrm{~s}$} & & \multicolumn{2}{c}{$t=14.5 \mathrm{~s}$} \\
\cline { 2 - 3 } \cline { 5 - 6 } & $\epsilon_{y}(\mathrm{~mm} \mathrm{mrad})$ & $\Delta \epsilon_{y}$ & & $\epsilon_{y}(\mathrm{~mm} \mathrm{mrad})$ & $\Delta \epsilon_{y}$ \\
\hline Two-scan & $1.59( \pm 0.02)$ & $\ldots$ & & $1.15( \pm 0.02)$ & $\ldots$ \\
One-scan T & $1.40( \pm 0.13)$ & $-11.95 \%$ & & $0.97( \pm 0.16)$ & $-15.7 \%$ \\
One-scan B & $1.45( \pm 0.10)$ & $-8.81 \%$ & & $0.97( \pm 0.17)$ & $-15.7 \%$ \\
\hline \hline
\end{tabular}

reflected by a more considerable difference in CDFs (Fig. 5). The magnitude of this emittance decrease, compared with for the vertical case, could be attributed to a significantly higher initial emittance.

Comparisons in the closed orbit showed a small change from $-4.05( \pm 0.04)$ to $-4.22( \pm 0.04) \mathrm{mm}$ during cooling, which could be explained by a change in the mean momentum offset of the beam, $\Delta \bar{\delta}$. This affects the mean horizontal position of the beam at the scraper through dispersion, $\Delta x_{0}=D_{x} \Delta \bar{\delta}$, and for this measurement corresponds to $\Delta \bar{\delta}=-1.2( \pm 0.3) \times 10^{-4}$. In the case that the mean momentum offset was more accurately known, it would be possible to use this relation to corroborate the value $D_{x}$. This would require the use of more Schottky pickups operating at a higher harmonic to give a better time resolution in the spectral density distribution.

A comparison of these measurements with simulations showed similar trends for the $t=14.5 \mathrm{~s}$ case as for the vertical measurement previously, with small deviations from Gaussian at the core and tails after cooling. However, for the earlier higher-momentum spread case, $t=7.8 \mathrm{~s}$, some discrepancy was observed in that the characteristic crossing

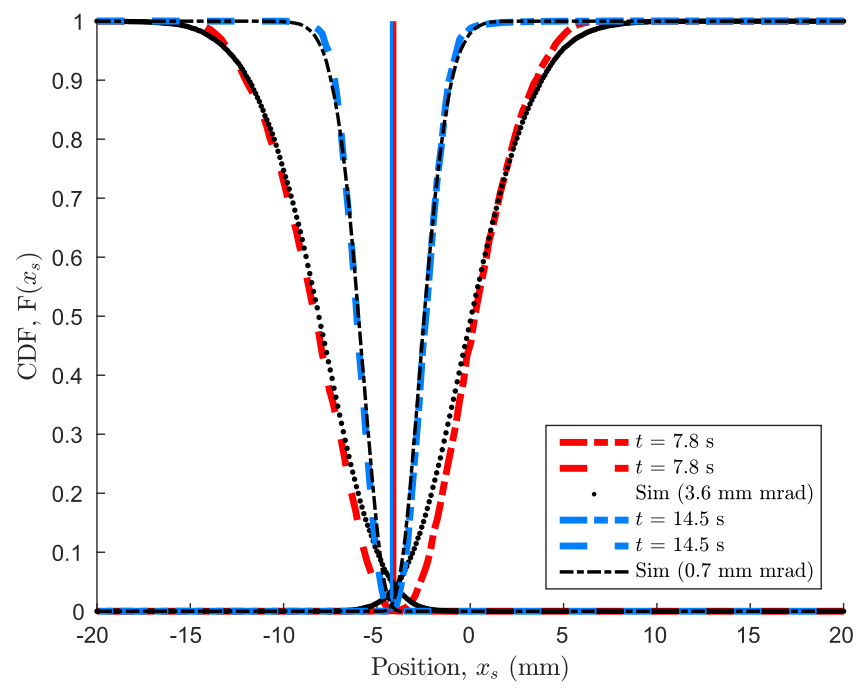

FIG. 5. Horizontal measured CDFs for scraper measurements at $650 \mathrm{keV}$. Calculated dispersion-dependent closed orbit values are displayed. of CDFs above $F_{ \pm}=0$ at the core of the beam (due to dispersive effects) was not observed. An example of such a crossing may be seen in Fig. 8. Further measurements at this energy would be required to determine the cause of this difference in simulated and measured distributions, since at the $100 \mathrm{keV}$ plateau a characteristic crossing above $F_{ \pm}=0$ was observed, as expected.

\section{B. Extraction $100 \mathrm{keV}$ energy plateau}

For this energy plateau, two sets of measurements $(2 \times 4$ directions) were made at the same cycle time, $t=28.9 \mathrm{~s}$, with the electron cooler on and off. This allows a comparison between an uncooled beam with one cooled for $3.9 \mathrm{~s}$.

Figure 6 shows the CDFs and corresponding simulation distributions for vertical reconstructed emittances of 0.53 $( \pm 0.01)$ and $2.55( \pm 0.03) \mathrm{mm}$ mrad in the presence of and without electron cooling, respectively. Comparisons with simulated Gaussian beams of the same emittance values show notable differences in the case of no cooling. A wider core could be explained by the IBS acting more significantly upon the beam core, since growth due to this effect is proportional to intensity. At $100 \mathrm{keV}$, the electron cooler has reduced $\epsilon_{y}$ by $79( \pm 2) \%$ of what it was without compensative measures, over $3.9 \mathrm{~s}$.

A good agreement between the Gaussian simulation and the cooling case was observed. This could be due to the beam quickly reaching equilibrium and having a reduced transverse size. This smaller transverse size results in the distribution "seeing" a more uniform electron velocity (at the center of the parabolic distribution of electron velocities). Continued cooling in this configuration leads to a Gaussian beam. It is also worth mentioning that, while for both vertical cases a similar negative offset was observed as for the $650 \mathrm{keV}$ measurements, between the two cases at

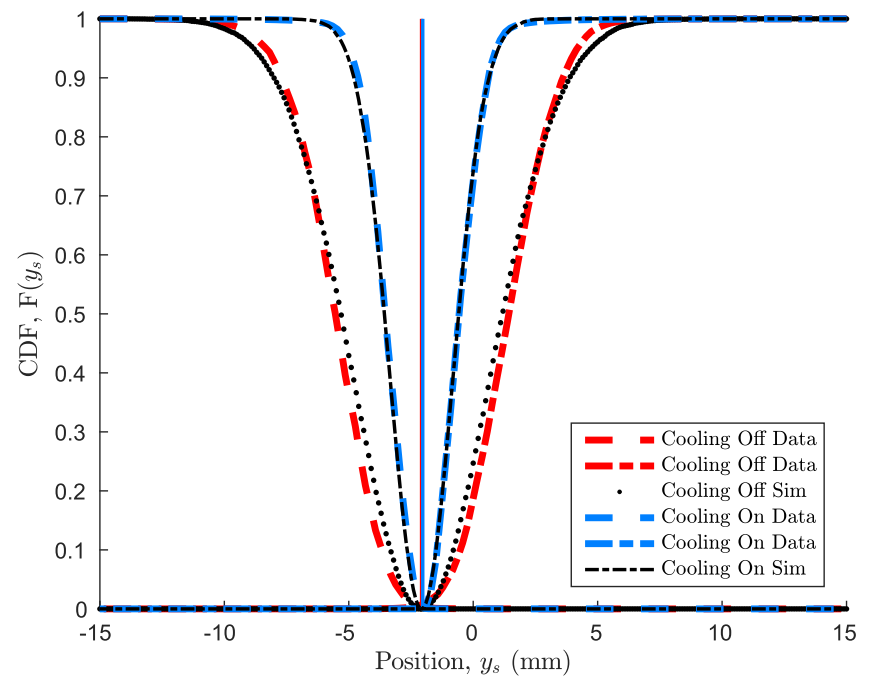

FIG. 6. CDFs produced from vertical scraper measurements at $100 \mathrm{keV}$ with overlaying Gaussian distributions generated from simulations. 
$100 \mathrm{keV}$ there was no significant change in the vertical closed orbit.

For the horizontal emittance at $100 \mathrm{keV}$, it was necessary to estimate the longitudinal momentum spread, since Schottky measurements were not available. Utilising the single-scan algorithm by taking $\sigma_{\delta}$ and $\epsilon_{x}$ as free parameters, fits were made to both scraper scan directions for the nocooling case. Taking the result with the best goodness of fit, a value of $\sigma_{\delta}=9.4( \pm 0.2) \times 10^{-4}$ was extracted. The fit may be seen in Fig. 7, with a slight deviation at the core, suggesting an underestimation. Although this method was shown to work with an accuracy value of around $10 \%$ for the vertical emittance measurement before cooling at $650 \mathrm{keV}$, the uncertainty on this value was increased to $\pm 1.9 \times 10^{-4}$ (corresponding to 20\%) to ensure the uncertain nature of this method carried through to the final reconstructed emittance value. For the case in the presence of cooling, the algorithm returned a values of the order $10^{-7}$ in both directions, so a value of $\sigma_{\delta}=0( \pm 2) \times 10^{-4}$ was estimated.

Utilizing the momentum spread values extracted from the single-scan algorithm, the $100 \mathrm{keV}$ horizontal measurements show an emittance difference of strikingly similar magnitude, $78( \pm 10) \%[2.5( \pm 0.2)$ and $0.55( \pm 0.04) \mathrm{mm} \mathrm{mrad}]$, to their vertical counterparts.

Simulations based on the reconstructed values, displayed in Fig. 8 alongside the measured CDFs, show good agreement for both cases, including the characteristic raised CDF crossing point typically related to dispersive effects. A small difference in the reconstructed closed orbit is observed, similarly for the $650 \mathrm{keV}$ case, corresponding to a value of $\Delta \bar{\delta}=1.7( \pm 0.3) \times 10^{-4}$. The simulation comparison also highlights an asymmetry in the noncooling CDFs, a characteristic observed previously when exploring the effects of a correlation between emittance and longitudinal momentum offset [12], which is investigated in the following section.

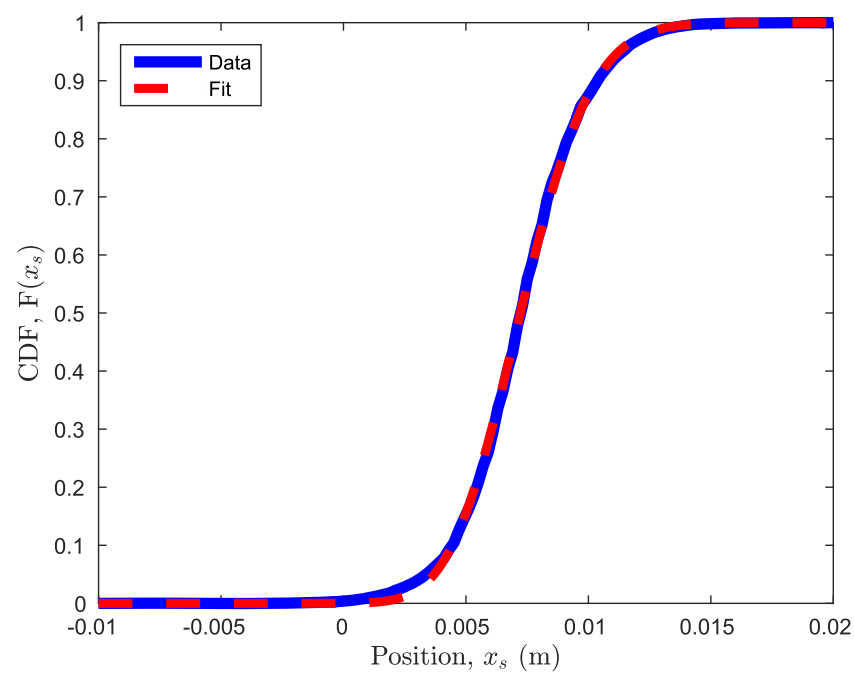

FIG. 7. Gaussian fit to a horizontal scraper scan at $100 \mathrm{keV}$, taking $\epsilon_{x}$ and $\sigma_{\delta}$ as free parameters.

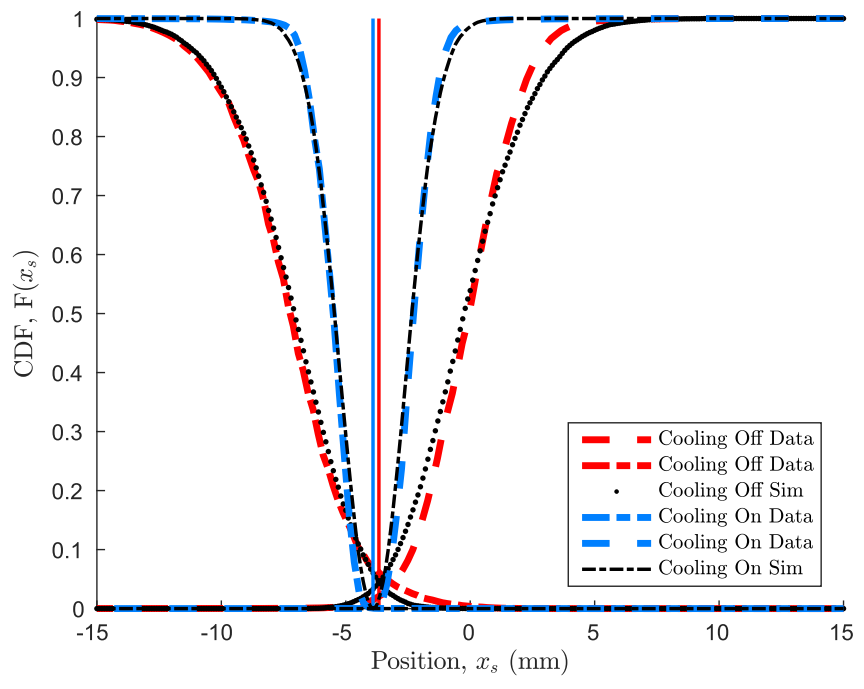

FIG. 8. CDFs extracted from horizontal scraper measurements at the $100 \mathrm{keV}$ cooling plateau. Simulations of CDFs corresponding to Gaussian beams with the measured emittances and momentum spreads are also displayed.

\section{Emittance-momentum offset correlation}

A quantity for determining a correlation between the emittance of individual particles in the beam and their longitudinal momentum offsets may be extracted from pairs of scraper measurements for coasting beams. Such a correlation could occur due to nonuniform transverse distribution in longitudinal electron velocities in the electron cooler beam. A parabolic transverse distribution of these velocities (with a minimum at the center of the beam) would result in ion beam particles with a higher maximum transverse amplitude having a higher (more positive) momentum offset than those at the core of the beam, after exposure to electron cooling. It should be noted that, although both effects are related through dependence on electron beam parameters, this effect is separate from the bi-Gaussian distribution causing effect, which depends on the nonuniform density of the electron beam. The effects may be considered separately in ELENA, since the emittance-momentum offset correlation is significant only in the presence of dispersion.

The extracted quantity was previously shown to accurately give a measure of the emittance-momentum offset correlation for bi-Gaussian beams through simulations [12]. Here the quantity is divided by $\sqrt{\epsilon_{x}}$ to normalize to the rms emittance of the beam. The normalized emittance-momentum spread correlation coefficient, henceforth here referred to as the correlation coefficient, may be expressed as and calculated from scraper measurements by

$$
\phi_{\epsilon \delta}=\langle(\delta-\bar{\delta} A)\rangle \epsilon_{x}^{-1 / 2}=\frac{\sigma_{+}^{2}-\sigma_{-}^{2}}{4 D_{x} \sqrt{\beta_{x} \epsilon_{x}}},
$$

here for the horizontal case, and similarly for when nonzero vertical dispersion is present. 
TABLE III. Extracted correlation coefficients for both horizontal measurements along both energy plateaus. The bottom row shows the absolute change between successive measurements.

\begin{tabular}{lccccc}
\hline \hline & \multicolumn{2}{c}{$650 \mathrm{keV}$} & & \multicolumn{2}{c}{$100 \mathrm{keV}$} \\
\cline { 2 - 3 } \cline { 5 - 6 } & $t=7.8$ & $t=14.5$ & & No cooling & Cooling \\
\hline$\phi_{\epsilon \delta}\left(\times 10^{-5}\right)$ & -5.4 & 1.0 & & -16 & 0.3 \\
Error $\phi_{\epsilon \delta}\left(\times 10^{-5}\right)$ & 2.5 & 1.1 & & 3.1 & 0.9 \\
$\Delta \phi_{\epsilon \delta}\left(\times 10^{-5}\right)$ & $6.4 \pm 3.6$ & & \multicolumn{2}{c}{$16.3 \pm 4$} \\
\hline \hline
\end{tabular}

Table III shows the results when calculating $\phi_{\epsilon \delta}$ for all horizontal measurements. Large uncertainties arise from larger uncertainties in $\epsilon_{x}$ and $\beta_{x}$ combined with uncertainties on the quantities $\sigma_{ \pm}$.

While it is difficult to take any immediate meaning from the absolute magnitude of a single value of $\phi_{\epsilon \delta}$, observations of the sign, \pm , and changes between successive measurements can give insight into how this effect evolves over time. Along the two ELENA plateaus, there is a clear positive change in $\phi_{\epsilon \delta}$, indicating that indeed, during cooling, particles at the core are gaining a less significant momentum offset than those at the tails.

An initial negative value of $\phi_{\epsilon \delta}$ would suggest that there already exists a negative emittance-momentum offset correlation at the start of both plateaus, perhaps brought about by the deceleration process, and that it may be unintentionally corrected for during cooling. Further measurements should be taken to investigate this possibility and to fully understand the relevant factors. It would be of particular interest to compare these results with measurements at different times for a coasting beam with no cooling present.

To ensure that this quantity is representative of some emittance-momentum offset correlation, values of $\phi_{\epsilon \delta}$ were calculated in the vertical plane. As there is negligible dispersion in this plane, it was expected that there would be no observable change in the coefficient. By inserting the same value of $D_{x}$ into $D_{y}$ in the equation (solely to negate division by zero), benchmarking values could be extracted from the vertical data. It should be explicitly stated that these values are calculated only for comparative reasons and in no way represent any physical quantities of the beam. The results are displayed in Table IV and confirm

TABLE IV. Correlation coefficients calculated for the vertical scraper measurements. Results displayed in this table are intended for benchmarking against the horizontal case only.

\begin{tabular}{lccccc}
\hline \hline & \multicolumn{2}{c}{$650 \mathrm{keV}$} & & \multicolumn{2}{c}{$100 \mathrm{keV}$} \\
\cline { 2 - 3 } \cline { 5 - 6 } & $t=7.8$ & $t=14.5$ & & No cooling & Cooling \\
\hline$\phi_{\epsilon \delta}\left(\times 10^{-5}\right)$ & 0.3 & -0.5 & & 0.5 & 0.7 \\
Error $\phi_{\epsilon \delta}\left(\times 10^{-5}\right)$ & 0.3 & 0.3 & & 0.2 & 0.2 \\
$\Delta \phi_{\epsilon \delta}\left(\times 10^{-5}\right)$ & $-0.7 \pm 0.6$ & & \multicolumn{2}{c}{$0.2 \pm 0.4$} \\
\hline \hline
\end{tabular}

that no significant change is observed, as expected. This suggests that these measurements give some insight into more complex beam behavior than has previously been possible through scraper measurements alone, and further investigations would be well founded.

\section{DISCUSSION}

Tables V and VI summarize all quantities extracted from the scraper measurements along both plateaus. Despite measurements being taken shortly after initial commissioning of the electron cooler, the emittance has been reduced in both planes at both energy plateaus. With these measurements, it is possible only to obtain a lower limit on the cooling rates, since is it likely that the beams had reached equilibrium earlier than the second scraper measurements.

A previous study, in which BETACOOL [16] was used to predict cooling rates in ELENA, showed results compatible with these measurements [17]. For comparison, in the simulations with initial emittances $\epsilon_{x, y}=2.8 \mathrm{~mm} \mathrm{mrad}$, final emittances of $\epsilon_{x}=0.52$ and $\epsilon_{y}=0.33 \mathrm{~mm} \mathrm{mrad}$ were achieved after $2 \mathrm{~s}$ of electron cooling at $100 \mathrm{keV}$.

Since IBS is proportional to $1 / \gamma^{4}$, it could be expected that the final (or equilibrium) emittances along the ejection plateau would be higher than those on the intermediate plateau. It is possible that this is not the case here, since the decelerating cycle was not yet optimized and larger losses were observed during the final deceleration ramp, leading to a lower-intensity beam and, hence, reduced collective effects. It is proposed that incremental measurements during a cooling plateau, while monitoring beam intensity,

TABLE V. Intermediate plateau summary table. Note: Changes in emittance are expressed as percentages of initial emittance.

\begin{tabular}{lrrr}
\hline \hline & \multicolumn{1}{c}{$t=7.8 \mathrm{~s}$} & \multicolumn{1}{c}{$t=14.5 \mathrm{~s}$} & \multicolumn{1}{c}{ Change } \\
\hline$\epsilon_{y}(\mathrm{~mm} \mathrm{mrad})$ & $1.59 \pm 0.02$ & $1.15 \pm 0.02$ & $28 \% \pm 2 \%$ \\
$y_{0}(\mathrm{~mm})$ & $-2.88 \pm 0.03$ & $-2.89 \pm 0.03$ & $-0.01 \pm 0.06$ \\
$\epsilon_{x}(\mathrm{~mm} \mathrm{mrad})$ & $3.6 \pm 0.27$ & $0.70 \pm 0.05$ & $81 \% \pm 10 \%$ \\
$x_{0}(\mathrm{~mm})$ & $-4.05 \pm 0.04$ & $-4.22 \pm 0.04$ & $-0.17 \pm 0.08$ \\
$\sigma_{\delta}\left(\times 10^{-3}\right)$ & $1.10 \pm 0.16$ & $0.22 \pm 0.03$ & $-0.88 \pm 0.19$ \\
$\phi_{\epsilon \delta}\left(\times 10^{-5}\right)$ & $-5.4 \pm 2.50$ & $1.00 \pm 1.10$ & $6.40 \pm 3.6$ \\
\hline \hline
\end{tabular}

TABLE VI. Ejection plateau summary table. "Off" and "on" refer to the status of the electron cooler. Note: Changes in emittance are expressed as percentages of initial emittance.

\begin{tabular}{lrrc}
\hline \hline & \multicolumn{1}{c}{ Off } & \multicolumn{1}{c}{ On } & Change \\
\hline$\epsilon_{y}(\mathrm{~mm} \mathrm{mrad})$ & $2.55 \pm 0.03$ & $0.53 \pm 0.01$ & $79 \% \pm 2 \%$ \\
$y_{0}(\mathrm{~mm})$ & $-2.08 \pm 0.03$ & $-2.03 \pm 0.03$ & $0.05 \pm 0.06$ \\
$\epsilon_{x}(\mathrm{~mm} \mathrm{mrad})$ & $2.5 \pm 0.20$ & $0.55 \pm 0.04$ & $78 \% \pm 10 \%$ \\
$x_{0}(\mathrm{~mm})$ & $-3.67 \pm 0.04$ & $-3.91 \pm 0.04$ & $-0.24 \pm 0.08$ \\
$\sigma_{\delta}\left(\times 10^{-4}\right)$ & $0.94 \pm 0.19$ & $0.0 \pm 0.2$ & $-0.94 \pm 0.39$ \\
$\phi_{\epsilon \delta}\left(\times 10^{-5}\right)$ & $-16 \pm 3.1$ & $0.3 \pm 0.9$ & $16.3 \pm 4$ \\
\hline \hline
\end{tabular}


could provide accurate estimations for the cooling rates and an insight into equilibrium emittance values under various conditions.

The different relative reduction in the horizontal and vertical emittances at the two energy plateaus could be related to the initial emittances before cooling, perhaps smaller vertical emittances coming from the $\mathrm{AD}$. Additionally, it could hint that vertical cooling at the intermediate plateau is not yet well optimized. Further measurements at the injection energy would provide some context for this observation.

From $\beta_{x, y}$ values at the electron cooler (Table I), estimations for horizontal beam widths encapsulating $\approx 99.7 \%$ of the beam $\left(3 \sigma_{r m s} \approx 3 \sqrt{\epsilon \beta}\right)$ give around $7.8 \pm 0.9$ and $6.9 \pm 0.9 \mathrm{~mm}$ for initial measured emittances on the 650 and $100 \mathrm{keV}$ plateaus, respectively. Transverse beam sizes here are certainly large enough to be nonuniformly affected by the parabolic distribution of electron velocities in the electron beam with nominal radius $25 \mathrm{~mm}$, supported by observations of $\phi_{\epsilon \delta}$.

An unexpected strong influence of dispersion leading to increased cooling rates seen in previous studies $[18,19]$ could certainly be explored further by utilizing a combination of $\phi_{\epsilon \delta}$ measurements while making variations in the $\beta_{x} / D_{x}$ ratio at the electron cooler. Additionally, changes to electron cooler parameters, such as the $e^{-}$beam energy, density, and, most importantly, the transverse radius, while monitoring this quantity would also be of great interest.

\section{SUMMARY AND OUTLOOK}

Measurements of the transverse statistical emittances during the 650 and $100 \mathrm{keV}$ plateaus of ELENA's deceleration cycle have been made, and the effects of electron cooling have been observed. The measurements were analyzed with two new scraper algorithms which were able to expose new information on beam quality, in particular, the emittance-momentum offset correlation coefficient $\phi_{\epsilon \delta}$. During this study, practical considerations for the future use of these algorithms have been highlighted, for example, the clipping of erroneous high-amplitude data or accounting for scraper "flight time" between activation and beam interception (Fig. 3), to allow for the most accurate results.

Acquired beam profiles have shown evidence of nonGaussian beam distributions due to the effects of electron cooling and, in the case of a coasting noncooled beam at the extraction plateau, due to the effects of IBS. Despite these non-Gaussian effects, the single-scan line-fit algorithm has been shown to perform well under these conditions and was used to estimate the momentum spread of the beam, the results of which were used in combination with the twoscan algorithm to determine the emittance in the presence of dispersion.

Finally, suggestions for future studies using these techniques have been proposed. In particular, numerous incremental measurements during the entire cycle of ELENA, coupled with extensive Schottky data, would be extremely beneficial in both the understanding of ELENA's beam evolution and the full capability of these new algorithms. It would serve well to compare estimates of the momentum spread made by the line-fit algorithm with Schottky data in more detail, as this could prove a useful tool in quick estimations of momentum spread. The potential gains from combining opposing scraper measurements are clear, with a particular emphasis on studying the evolution of $\phi_{\epsilon \sigma}$ in more detail, in ELENA and other machines with comparable challenges and compatible hardware.

\section{ACKNOWLEDGMENTS}

We thank all of our colleagues at CERN involved in the ELENA project, in particular, Tommy Eriksson and Gerard Tranquille for insightful discussions around the electron cooler. This work is supported by the EU under Grant Agreement No. 624854 and the STFC Cockcroft Institute core Grant No. ST/G008248/1.

[1] Design study of the antiproton decelerator: AD, edited by S. Maury, Report No. CERN/PS 96-43 (AR), 1996.

[2] S. Maury, The antiproton decelerator: AD, Hyperfine Interact. 109, 43 (1997).

[3] Extra low energy antiproton ring (ELENA) and its transfer lines, edited by V. Chohan et al., Report No. CERN-2014002, 2014.

[4] S. Maury, W. Oelert, W. Bartmann, P. Belochitskii, H. Breuker, F. Butin, C. Carli, T. Eriksson, S. Pasinelli, and G. Tranquille, ELENA: The extra low energy anti-proton facility at CERN, Hyperfine Interact. 229, 105 (2014).

[5] T. Eriksson, W. Bartmann, P. Belochitskii, L. Bojtár, H. Breuker, F. Butin, C. Carli, B. Dupuy, P. Freyermuth, and L. Jørgensen, ELENA-From installation to commissioning, in Proceedings of the 8th International Particle Accelerator Conference (IPAC2017), Copenhagen, Denmark (JACoW, Geneva, 2017).

[6] M. Martini, Intrabeam scattering in the ACOL-AA machines, Report No. CERN-PS-AA-84-9, 1984.

[7] A. Piwinski, Intra-beam scattering, in Proceedings of the 9th International Conference on High Energy Accelerators, Stanford, CA (SLAC, Stanford, 1974), p. 405.

[8] J. Bjorken and S. Mtingwa, Intrabeam scattering, Part. Accel. 13, 115 (1983).

[9] A. Piwinski, Intra-beam scattering, Report No. CERN-9201, 1992, p. 226.

[10] G. Tranquille, J. Cenede, A. Frassier, L. V. Jørgensen, A. Kolehmainen, B. Moles, and M. Timmins, The ELENA electron cooler, in Proceedings of the 7th International Particle Accelerator Conference (IPAC2016) (JACoW, Geneva, 2016), TUPMR006.

[11] V. Chohan et al., Beam measurement systems for the CERN antiproton decelerator (AD), in Proceedings of the 19th Particle Accelerator Conference, Chicago, IL, 2001 (IEEE, Piscataway, NJ, 2001). 
[12] J. R. Hunt, C. Carli, J. Resta-López, and C. P. Welsch, Emittance measurements in low energy ion storage rings, Nucl. Instrum. Methods Phys. Res., Sect. A 896, 139 (2018).

[13] O. Marqversen, M. E. Angoletta, M. Jaussi, R. MarcoHernandez, J. Molendijk, R. Ruffieux, J. Sanchez-Quesada, and L. Søby, The orbit measurement system for the CERN extra low energy antiproton ring, in Proceedings of the 6th International Beam Instrumentation Conference (IBIC2017) (JACoW, Geneva, 2018), TUPCF05.

[14] http://mad.web.cern.ch/mad/.

[15] http://www.python.org.

[16] A. Sidorin, I. N. Meshkov, I. A. Seleznev, A. V. Smirnov, E. M. Syresin, and G. V. Trubnikov, BETACOOL program for simulation of beam dynamics in storage rings, Nucl. Instrum. Methods Phys. Res., Sect. A 558, 325 (2006).

[17] J. Resta-López, J. R. Hunt, O. Karamyshev, and C. P. Welsch, Simulation studies of the beam cooling process in presence of heating effects in the extra low energy antiproton ring (ELENA), J. Instrum. 10, P05012 (2015).

[18] J. Bosser, C. Carli, M. Chanel, L. Marie, D. Möhl, and G. Tranquille, On the optimum dispersion of a storage ring for electron cooling with high space charge, Nucl. Instrum. Methods Phys. Res., Sect. A 441, 60 (2000).

[19] J. Bosser, C. Carli, M. Chanel, S. Maury, D. Möhl, and G. Tranquille, Optimum lattice functions for electron cooling, Nucl. Instrum. Methods Phys. Res., Sect. A 532, 422 (2004). 